\title{
LUSZTIG LIMIT OF QUANTUM SL(2) AT ROOT OF UNITY AND FUSION OF (1,P) VIRASORO LOGARITHMIC MINIMAL MODELS
}

\author{
P.V. BUSHLANOV, B.L. FEIGIN, A.M. GAINUTDINOV AND I.YU. TIPUNIN
}

\begin{abstract}
We introduce a Kazhdan-Lusztig-dual quantum group for $(1, p)$ Virasoro logarithmic minimal models as the Lusztig limit of the quantum $s \ell(2)$ at $p$ th root of unity and show that this limit is a Hopf algebra. We calculate tensor products of irreducible and projective representations of the quantum group and show that these tensor products coincide with the fusion of irreducible and logarithmic modules in the $(1, p)$ Virasoro logarithmic minimal models.
\end{abstract}

\section{INTRODUCTION}

Logarithmic conformal field theories most naturally appear as a scaling limit of twodimensional nonlocal lattice models at a critical point [1] and in quantum chains with a nondiagonalizable Hamiltonian [2]. Generally speaking, a conformal field theory appearing at the limit depends on a way of taking the limit and on chosen boundary conditions. The recent investigations [3, 4, 5] argue that for proper choice of boundary conditions the lattice models [1] give in a scaling limit logarithmic conformal models $\mathcal{W} \mathcal{L} \mathcal{M}(p, q)$ with the triplet $\mathcal{W}_{p, q}$-algebra of symmetry introduced in [6] and in [7, 8] for $q=1$. The chiral algebra in these conformal models is an extension of the vacuum module of the Virasoro algebra $\mathcal{V}_{p, q}$ with the central charge $c_{p, q}=13-6 p / q-6 q / p$ by the triplet of the Virasoro primary fields with conformal dimension $\Delta_{1,3}$.

The most investigated models are those with $q=1$. In this case, the conformal field theories $\mathcal{W} \mathcal{L} \mathcal{M}(1, p)$ corresponding to the lattice models were described in terms of symplectic fermions in [9] (for $p=2$ ) and were studied in numerous papers [10, 11, 12, 8, 13, 14, 15, 16, 17, 18, 27, 28]. For this set of models, the representation categories of the triplet algebra $\mathcal{W}_{p}$ and of the finite-dimensional quantum group $\overline{\mathcal{U}}_{\mathfrak{q}} s \ell(2)$ [19] at the $p$ th root of unity are equivalent as braided tensor categories [20]. This is the manifestation of the Kazhdan-Lusztig duality between vertex-operator algebras and quantum groups in logarithmic models. This duality means that (i) there is a one-to-one correspondence between representations; (ii) fusion rules of a conformal model can be calculated by tensor products of a quantum group representations and (iii) the modular group action generated from chiral characters coincides with the one on the center of the corresponding quantum group. In the logarithmic models $\mathcal{W} \mathcal{L} \mathcal{M}(1, p)$, the Kazhdan-Lusztig duality is presented in its full strength (see also review [21]). In particular, the fusion calculated in [8] (see also [29]) coincides with the Grothendieck ring of the $\overline{\mathcal{U}}_{\mathfrak{q}} s \ell(2)$. 
For general coprime $p$ and $q$, the models $\mathcal{W} \mathcal{L} \mathcal{M}(p, q)$ also demonstrate the KazhdanLusztig duality with a quantum group [6] but relation between the quantum group and the $\mathcal{W}_{p, q}$ algebra is more subtle. There is no one-to-one correspondence between representations but the modular group action on the center [22] coincides with the one on chiral characters in the $\mathcal{W}_{p, q}$ theory and the quantum-group fusion [6] coincides with the fusion [23] of $\mathcal{W}_{p, q}$ representations under the identification of the fusion generators $\mathcal{K}_{1,2}^{+} \rightarrow(1,2)_{\mathcal{W}}$ and $\mathcal{K}_{2,1}^{+} \rightarrow(2,1)_{\mathcal{W}}$; we also identify $\mathcal{K}_{a, b}^{+} \rightarrow(a, b)_{\mathcal{W}}, \mathcal{K}_{a, b}^{-} \rightarrow\left(\Delta_{a, 3 q-b}\right)_{\mathcal{W}} 1$. This fusion was also derived [24] from the modular group properties of the chiral characters.

Other choice of boundary conditions in the lattice models [1] leads to logarithmic conformal field models $\mathcal{L} \mathcal{M}(p, q)$ with the Virasoro symmetry $\mathcal{V}_{p, q}$. Fusion rules for these models were calculated in [25] using a lattice approach and for some cases in [26, 36] using the Nahm algorithm and in [2] using quantum-group symmetries in XXZ models at a root of unity.

In the present paper, we propose using the Kazhdan-Lusztig duality in calculating the fusion rules for the simplest subset $\mathcal{L} \mathcal{M}(1, p)$ of the $\mathcal{L} \mathcal{M}(p, q)$ models. We construct a quantum group dual to the Virasoro algebra $\mathcal{V}_{p}$ as an extension of the quantum group $\overline{\mathcal{U}}_{\mathfrak{q}} s \ell(2)$ dual to $\mathcal{W}_{p}$ from the $\mathcal{W} \mathcal{L} \mathcal{M}(1, p)$ models. This quantum group is the Lusztig limit $\mathcal{L} \mathcal{U}_{\mathfrak{q}} s \ell(2)$ of the usual quantum $s \ell(2)$ as $\mathfrak{q} \rightarrow e^{\imath \pi / p}$ and has the set of irreducible representations $X_{s, r}^{\alpha}$, where $s=1,2, \ldots, p$ and $\alpha= \pm$ are $\overline{\mathcal{U}}_{\mathfrak{q}} s \ell(2)$ highest weight parameters and $\frac{r-1}{2}, r \in \mathbb{N}$, is the $s \ell(2)$ spin (see precise definitions in Sec. 3.1). The module $X_{s, r}^{\alpha}$ is a tensor product of $s$-dimensional irreducible $\overline{\mathcal{U}}_{\mathfrak{q}} s \ell(2)$ - and $r$-dimensional irreducible $s \ell(2)$-modules. To each $X_{s, r}^{\alpha}$, a projective cover $\mathcal{P}_{s, r}^{\alpha}$ corresponds and $\mathcal{P}_{p, r}^{\alpha}=X_{p, r}^{\alpha}$. The set of irreducible and projective modules is closed under tensor products.

We show that the fusion [25] of irreducible and logarithmic $\mathcal{V}_{p}$-representations coincides with tensor products of $\mathcal{L} \mathcal{U}_{\mathfrak{q}} s \ell(2)$ irreducible and projective modules. To formulate the main result of the paper, we introduce the following sum notations

$$
\begin{gathered}
\bigoplus_{r=a}^{b} f(r)=\bigoplus_{r=a}^{b}\left(1-\frac{1}{2} \delta_{r, a}-\frac{1}{2} \delta_{r, b}\right) f(r), \\
\bigoplus_{r=a}^{b} f(r)=\bigoplus_{r=a}^{b}\left(1-\frac{3}{4} \delta_{r, a}-\frac{1}{4} \delta_{r, a+2}\left(1+\delta_{a,-1}\right)-\frac{1}{4} \delta_{r, b-2}-\frac{3}{4} \delta_{r, b}\right) f(r) .
\end{gathered}
$$

1 Indecomposable rank-2 and rank-3 representations appearing in the fusion [23] are identified with the corresponding quantum-group modules [22] in the following way: $\mathcal{P}_{a, b}^{+,+} \rightarrow\left(\mathcal{R}_{p, b}^{p-a, 0}\right)_{\mathcal{W}}$, $\mathcal{P}_{a, b}^{+,-} \rightarrow\left(\mathcal{R}_{a, q}^{0, q-b}\right)_{\mathcal{W}}$ and $\mathrm{P}_{a, b}^{+} \rightarrow\left(\mathcal{R}_{p, q}^{p-a, q-b}\right)_{\mathcal{W}}, \mathrm{P}_{a, b}^{-} \rightarrow\left(\mathcal{R}_{2 p, q}^{p-a, q-b}\right)_{\mathcal{W}}$ 
1.1. Theorem. The tensor products between irreducible $\mathcal{L} \mathfrak{U}_{\mathfrak{q}} s \ell(2)$-modules are

$$
X_{s_{1}, r_{1}}^{\alpha} \otimes X_{s_{2}, r_{2}}^{\beta}=\bigoplus_{\substack{r=\left|r_{1}-r_{2}\right|+1 \\ \text { step }=2}}^{r_{1}+r_{2}-1}\left(\bigoplus_{\substack{s=\left|s_{1}-s_{2}\right|+1 \\ \text { step }=2}}^{\substack{\min \left(s_{1}+s_{2}-1, 2 p-s_{1}-s_{2}-1\right)}} X_{s, r}^{\alpha \beta}+\bigoplus_{\substack{s=2 p-s_{1}-s_{2}+1 \\ \text { step }=2}}^{p-\gamma_{2}} \mathcal{P}_{s, r}^{\alpha \beta}\right)
$$

between the irreducible and projective modules are

$$
X_{s_{1}, r_{1}}^{\alpha} \otimes \mathcal{P}_{s_{2}, r_{2}}^{\beta}=\bigoplus_{\substack{r=\left|r_{1}-r_{2}\right|+1 \\ \text { step }=2}}^{r_{1}+r_{2}-1}\left(\bigoplus_{\substack{s=\left|s_{1}-s_{2}\right|+1 \\ \text { step=2 }}}^{\substack{\min \left(s_{1}+s_{2}-1, 2 p-s_{1}-s_{2}-1\right)}} \mathcal{P}_{\substack{s=2 p-s_{1}-s_{2}+1 \\ \text { step }=2}}^{\alpha \beta}+2 \bigoplus_{\substack{s, r \\ \text { ste }}}^{\alpha-\gamma_{2}}+2 \bigoplus_{\substack{r=\left|r_{1}-r_{2}\right| \\ \text { step }=2}}^{r_{1}+r_{2}} \bigoplus_{\substack{s=s_{1}+s_{2}+1 \\ \text { step }=2}}^{p-\gamma_{1}} \mathcal{P}_{s, r}^{-\alpha \beta},\right.
$$

and between the projective modules are

$$
\begin{aligned}
& \mathcal{P}_{s_{1}, r_{1}}^{\alpha} \otimes \mathcal{P}_{s_{2}, r_{2}}^{\beta}=2 \bigoplus_{\substack{r=\left|r_{1}-r_{2}\right|+1 \\
\text { step }=2}}^{r_{1}+r_{2}-1}\left(\bigoplus_{\substack{s=\left|s_{1}-s_{2}\right|+1 \\
\text { step=2 }}}^{\substack{\min \left(s_{1}+s_{2}-1 \\
2 p-s_{1}-s_{2}-1\right)}} \mathcal{P}_{s, r}^{\alpha \beta}+2 \bigoplus_{\substack{s=2 p-s_{1}-s_{2}+1 \\
\text { step }=2}}^{p-\gamma_{2}} \mathcal{P}_{s, r}^{\alpha \beta}\right) \\
& +2 \bigoplus_{\substack{r=\left|r_{1}-r_{2}\right| \\
\text { step }=2}}^{r_{1}+r_{2}}\left(\bigoplus_{\substack{s=\left|p-s_{1}-s_{2}\right|+1 \\
\text { step }=2}}^{\substack{\min \left(p-s_{1}+s_{2}-1, p+s_{1}-s_{2}-1\right)}} \mathcal{P}_{s, r}^{-\alpha \beta}+2 \bigoplus_{\substack{s=\min \left(p-s_{1}+s_{2}+1, r \\
p+s_{1}-s_{2}+1\right)}}^{p-\gamma_{1}} \mathcal{P}_{s, r}^{-\alpha \beta}\right)+4 \bigoplus_{\substack{r=\left|r_{1}-r_{2}\right|-1 \\
\text { step }=2}}^{r_{1}+r_{2}+1} \bigoplus_{\substack{s=s_{1}+s_{2}+1 \\
\text { step }=2}}^{p-\gamma_{2}} \mathcal{P}_{s, r}^{\alpha \beta},
\end{aligned}
$$

where we set $\gamma_{1}=\left(s_{1}+s_{2}+1\right) \bmod 2, \gamma_{2}=\left(s_{1}+s_{2}+p+1\right) \bmod 2$.

The $\mathcal{L} \mathcal{U}_{\mathfrak{q}} s \ell(2)$ representation category $\mathcal{C}_{p}$ is a direct sum of two full subcategories $\mathcal{C}_{p}=$ $\mathrm{C}_{p}^{+} \oplus \mathrm{C}_{p}^{-}$such that there are no morphisms between $\mathrm{C}_{p}^{+}$and $\mathrm{C}_{p}^{-}$and the subcategory $\mathrm{C}_{p}^{+}$is closed under tensor products. The set of irreducible modules belonging to the subcategory $\mathcal{C}_{p}^{+}$is exhausted by the irreducible modules $X_{s, r}^{\alpha}$ with $\alpha=+$ whenever $r$ is $o d d$, and $\alpha=-$ whenever $r$ is even.

The category $\mathrm{C}_{p}^{+}$is equivalent as a tensor category to the category of Virasoro algebra representations appearing in $\mathcal{L} \mathcal{M}(1, p)$. We do not describe here this Virasoro category but note only that under this equivalence irreducible and projective modules are identified in the following way

$$
\begin{gathered}
X_{p, 2 r-1}^{+} \rightarrow \mathcal{R}_{2 r-1}^{0}, \quad X_{p, 2 r}^{-} \rightarrow \mathcal{R}_{2 r}^{0}, \quad \mathcal{P}_{s, 2 r-1}^{+} \rightarrow \mathcal{R}_{2 r-1}^{p-s}, \quad \mathcal{P}_{p-s, 2 r}^{-} \rightarrow \mathcal{R}_{2 r}^{s}, \\
x_{s, 2 r-1}^{+} \rightarrow(2 r-1, s), \quad X_{s, 2 r}^{-} \rightarrow(2 r, s), \quad 1 \leqslant s \leqslant p, \quad r \geqslant 1,
\end{gathered}
$$

where $(r, s)$ are the irreducible Virasoro modules with the heighest weights $\Delta_{r, s}=((p r-$ $\left.s)^{2}-(p-1)^{2}\right) / 4 p$ and the $\mathcal{R}_{r}^{s}$ are logarithmic Virasoro modules from $\mathcal{L} \mathcal{M}(1, p)$. Under this identification, the fusion [25] for $\mathcal{L M}(1, p)$ is given by tensor products of the corresponding $\mathcal{L} \mathcal{U}_{\mathfrak{q}} s \ell(2)$ representations.

The quantum groups dual to the logarithmic conformal models $\mathcal{L} \mathcal{M}(1, p)$ as well as $\mathcal{W L M}(1, p)$ can be constructed in the free field approach [19, 6]. In this approach, the 
corresponding quantum groups and the chiral algebras are mutual maximal centralizers of each other. To construct the quantum group for $\mathcal{L} \mathcal{M}(1, p)$, we first note that the chiral algebras $\mathcal{W}_{p}$ realized in the $\mathcal{W} \mathcal{L} \mathcal{M}(1, p)$ models admit $S L(2)$-action by symmetries. Invariants of this action is the universal enveloping of the Virasoro algebra $\mathcal{V}_{p}$ with the central charge $c_{p}=13-6 p-6 / p$. This suggests that a quantum group $\mathcal{L} \mathcal{U}_{\mathfrak{q}} s \ell(2)$ dual to the Virasoro algebra from $\mathcal{L} \mathcal{M}(1, p)$ should be a combination of the quantum group $\overline{\mathcal{U}}_{\mathfrak{q}} s \ell(2)$ dual to $\mathcal{W}_{p}$ and ordinary $s \ell(2)$.

The quantum group $\mathcal{L} \mathcal{U}_{\mathfrak{q}} s \ell(2)$ is constructed in the free-field approach using the screening operators of the Virasoro algebra $\mathcal{V}_{p}$ with the central charge $c_{p}$. We recall there are two screening operators $e=\oint e^{\sqrt{2 p} \varphi(z)} d z$ and $F=\oint e^{-\sqrt{\frac{2}{p}} \varphi(z)} d z$ commuting with $\mathcal{V}_{p}$ and the screening $F$ commutes [8] with the extended chiral algebra $\mathcal{W}_{p}$ and generates the lower-triangular part of the $\overline{\mathcal{U}}_{\mathfrak{q}} s \ell(2)$ with the relation $F^{p}=0$. Then, considering the deformation $F_{\epsilon}=\oint e^{\left(-\sqrt{\frac{2}{p}}+\epsilon\right) \varphi(z)} d z$, we can construct an operator $f=\lim _{\epsilon \rightarrow 0} \frac{F_{\epsilon}^{p}}{\epsilon}$. The operators $e$ and $f$ generate the $s \ell(2)$ from the previous paragraph. To obtain a Hopf-algebra structure on $\mathcal{L} \mathcal{U}_{\mathfrak{q}} s \ell(2)$, we use here the purely algebraic approach following Lusztig. We construct the quantum group $\mathcal{L} \mathcal{U}_{\mathfrak{q}} s \ell(2)$ as a limit of the quantum group $U_{\mathfrak{q}}(s \ell(2))$ as $\mathfrak{q} \rightarrow e^{\frac{2 \pi}{p}}$. There is an evident limit in which $E^{p}, F^{p}$ and $K^{p}$ become central but we consider another limit in which the relations $E^{p}=F^{p}=0, K^{2 p}=1$ are imposed but the generators $e=\frac{E^{p}}{[p] !}$ and $f=\frac{F^{p}}{[p] !}$ are kept in the limit. In the limit $\mathfrak{q} \rightarrow e^{\frac{2 \pi}{p}}$, we have $[p] !=0$ and the ambiguity $\frac{0}{0}$ is solved in such a way that the $e$ and $f$ become generators of the ordinary $s \ell(2)$. We thus obtain a Hopf algebra $\mathcal{L} \mathcal{U}_{\mathfrak{q}} s \ell(2)$ that contains the quantum group $\overline{\mathcal{U}}_{\mathfrak{q}} s \ell(2)$ as a Hopf ideal and the quotient is the $U(s \ell(2))$, the universal enveloping of the $s \ell(2)$.

It is noteworthy to mention that a similar duality is also presented in quantum spin chains (XXZ models) with nondiagonalizable action of the Hamiltonian. Here, there are two commuting actions of a Temperley-Lieb algebra (or a proper its extension) and of a quantum group. In other words, the space of spin states is a bimodule over the TemperleyLieb algebra (or its extension) and the corresponding quantum group [2]. Moreover, the quantum group symmetries are stable with respect to increasing the number of sites and should be kept in a scaling limit; fusion rules for Temperley-Lieb algebra representations are obtained by an induction procedure joining two chains end to end and can be calculated using only the quantum group symmetries [2, 30]. Thus, these two algebraic objects are in some duality which is a lattice version of the Kazhdan-Lusztig duality.

We also note that tensor products of $\mathcal{L} \mathcal{U}_{\mathfrak{q}} s \ell(2)$ projective modules reproduce the Virasoro fusion rules proposed in [2] under the identifications:

$$
\begin{array}{lllll}
p=2: & \mathcal{P}_{1,2 r-1}^{+} \rightarrow \mathcal{R}_{2 r-1}, & \mathcal{P}_{1,2 r}^{-} \rightarrow \mathcal{R}_{2 r}, & r \in \mathbb{N}, \\
p=3: & X_{3,2 r-1}^{+} \rightarrow \mathcal{R}_{3 r-2}, & \mathcal{P}_{1,2 r-1}^{+} \rightarrow \mathcal{R}_{3 r-1}, & \mathcal{P}_{2,2 r}^{-} \rightarrow \mathcal{R}_{3 r}, & r \in \mathbb{N} .
\end{array}
$$


The paper is organized as follows. In Sec. 2, we introduce the quantum group $\mathcal{L} \mathcal{U}_{\mathfrak{q}} s \ell(2)$ dual to the Virasoro algebra $\mathcal{V}_{p}$ and describe a Hopf algebra structure on $\mathcal{L} \mathcal{U}_{\mathfrak{q}} s \ell(2)$. In Sec. 3, we describe irreducible representations of $\mathcal{L} \mathcal{U}_{\mathfrak{q}} s \ell(2)$, calculate all possible extensions between them and then construct projective modules. In Sec. 4 , we decompose tensor products between irreducible and projective $\mathcal{L} \mathcal{U}_{\mathfrak{q}} s \ell(2)$-modules.

\section{QUANTUM GROUPS AS CENTRALIZERS OF VOAS.}

In this section, we introduce a quantum group that commutes with the Virasoro algebra $\mathcal{V}_{p}$ on the chiral space of states in the free massless scalar field theory

$$
\varphi(z) \varphi(w)=\log (z-w)
$$

with the energy-momentum tensor

$$
T=\frac{1}{2} \partial \varphi \partial \varphi+\frac{\alpha_{0}}{2} \partial^{2} \varphi
$$

where the background charge $\alpha_{0}=\alpha_{+}+\alpha_{-}=\sqrt{2 p}-\sqrt{2 / p}$. This quantum group is denoted as $\mathcal{L} \mathcal{U}_{\mathfrak{q}} s \ell(2)$ and constructed as some extension of the finite-dimensional quantum group $\overline{\mathcal{U}}_{\mathfrak{q}} s \ell(2)$ which is the maximal centralizer of the triplet algebra $\mathcal{W}_{p}$. We recall that $\mathcal{W}_{p}$ is an extension of $\mathcal{V}_{p}$ by the $s \ell(2)$-triplet of the fields $W^{ \pm, 0}(z)[8]$ :

$$
W^{-}(z):=\mathrm{e}^{-\alpha_{+} \varphi}(z), \quad W^{0}(z):=\left[S_{+}, W^{-}(z)\right], \quad W^{+}(z):=\left[S_{+}, W^{0}(z)\right],
$$

where $S_{+}$is the "long screening" operator $\oint \mathrm{e}^{\alpha_{+} \varphi} d z$. These three fields are Virasoro primaries and their conformal dimensions equal to $(2 p-1)$. The field $W^{-}(z)$ is the lowestweight vector (with respect to the Cartan $s \ell(2)$-generator $h=\frac{1}{\alpha_{+}} \varphi_{0}$ and $\varphi_{0}$ is the zeromode of $\partial \varphi(z))$, the field $W^{0}(z)$ has the $h$-weight equals to 0 and $W^{+}(z)$ is the highestweight vector of the $s \ell(2)$-triplet.

Irreducible representations of the triplet algebra $\mathcal{W}_{p}$ admit two commuting actions, $s \ell(2)$ - and $\mathcal{V}_{p}$-actions [6], and the Virasoro algebra $\mathcal{V}_{p}$ is the invariant of the $s \ell(2)$ action in the vacuum representation of $\mathcal{W}_{p}$. This suggests a construction of the maximal centralizer for $\mathcal{V}_{p}$ as an extension of the centralizer $\overline{\mathcal{U}}_{\mathfrak{q}} s \ell(2)$ for the triplet algebra $\mathcal{W}_{p}$ by the $s \ell(2)$ triplet: $e=S_{+}, h=\frac{1}{\alpha_{+}} \varphi_{0}$, and a "conjugate" operator $f$ to the long screening $S_{+}$.

2.1. The centralizer of $\mathcal{W}_{p}$. We recall the definition of the quantum group $\overline{\mathcal{U}}_{\mathfrak{q}} s \ell(2)$ [19] that commutes with the triplet algebra $\mathcal{W}_{p}$ action on the chiral space of states. The $\overline{\mathcal{U}}_{\mathfrak{q}} s \ell(2)$ can be constructed as the Drinfeld double of the Hopf algebra generated by the "short screening" operator $F=\oint \mathrm{e}^{\alpha_{-} \varphi(z)} d z$ and $K=\mathrm{e}^{-i \pi \alpha_{-} \varphi_{0}}$. The Hopf algebra structure is found from the action of these operators on fields. In particular, the comultiplication is calculated from the action of $F$ and $K$ on operator product expansions of fields. Details of constructing $\overline{\mathcal{U}}_{\mathfrak{q}} s \ell(2)$ are given in [19]. 
The quantum group $\overline{\mathcal{U}}_{\mathfrak{q}} s \ell(2)$ is the "restricted" quantum $s \ell(2)$ with $\mathfrak{q}=e^{i \pi / p}$ and the three generators $E, F$, and $K$ satisfying the standard relations for the quantum $s \ell(2)$,

$$
K E K^{-1}=\mathfrak{q}^{2} E, \quad K F K^{-1}=\mathfrak{q}^{-2} F, \quad[E, F]=\frac{K-K^{-1}}{\mathfrak{q}-\mathfrak{q}^{-1}},
$$

with some additional constraints,

$$
E^{p}=F^{p}=0, \quad K^{2 p}=\mathbf{1},
$$

and the Hopf-algebra structure is given by

$$
\begin{gathered}
\Delta(E)=\mathbf{1} \otimes E+E \otimes K, \quad \Delta(F)=K^{-1} \otimes F+F \otimes \mathbf{1}, \quad \Delta(K)=K \otimes K, \\
S(E)=-E K^{-1}, \quad S(F)=-K F, \quad S(K)=K^{-1}, \\
\epsilon(E)=\epsilon(F)=0, \quad \epsilon(K)=1 .
\end{gathered}
$$

The quantum group $\overline{\mathcal{U}}_{\mathfrak{q}} s \ell(2)$ admits $(p-1)$ dimensional family of inequivalent extensions by $s \ell(2)$ algebras acting on $\overline{\mathcal{U}}_{\mathfrak{q}} s \ell(2)$ as exterior derivatives [31]. There is an extension that admits a Hopf algebra structure to be defined in the following subsection.

2.2. The centralizer of $\mathcal{V}_{p}$. Here, we define a quantum group $\mathcal{L} \mathcal{U}_{\mathfrak{q}} s \ell(2)$ (i.e. a Hopf algebra) that commutes with the Virasoro algebra $\mathcal{V}_{p}$ on the chiral space of states.

2.2.1. Definition. The Hopf-algebra structure on $\mathcal{L} \mathcal{U}_{\mathfrak{q}} s \ell(2)$ is the following. The defining relations between the $E, F$, and $K$ generators are the same as in $\overline{\mathcal{U}}_{\mathfrak{q}} s \ell(2)$ and given in (2.2) and (2.3), and the usual $s \ell(2)$ relations between the $e, f$, and $h$ :

$$
[h, e]=e, \quad[h, f]=-f, \quad[e, f]=2 h,
$$

and the "mixed" relations

$$
\begin{aligned}
{[h, K]=0, \quad[E, e] } & =0, \quad[K, e]=0, \quad[F, f]=0, \quad[K, f]=0, \\
{[F, e] } & =\frac{1}{[p-1] !} K^{p} \frac{\mathfrak{q} K-\mathfrak{q}^{-1} K^{-1}}{\mathfrak{q}-\mathfrak{q}^{-1}} E^{p-1}, \\
{[E, f] } & =\frac{(-1)^{p+1}}{[p-1] !} F^{p-1} \frac{\mathfrak{q} K-\mathfrak{q}^{-1} K^{-1}}{\mathfrak{q}-\mathfrak{q}^{-1}}, \\
{[h, E] } & =\frac{1}{2} E A, \quad[h, F]=-\frac{1}{2} A F,
\end{aligned}
$$

where

$$
A=\sum_{s=1}^{p-1} \frac{\left(u_{s}\left(\mathfrak{q}^{-s-1}\right)-u_{s}\left(\mathfrak{q}^{s-1}\right)\right) K+\mathfrak{q}^{s-1} u_{s}\left(\mathfrak{q}^{s-1}\right)-\mathfrak{q}^{-s-1} u_{s}\left(\mathfrak{q}^{-s-1}\right)}{\left(\mathfrak{q}^{s-1}-\mathfrak{q}^{-s-1}\right) u_{s}\left(\mathfrak{q}^{-s-1}\right) u_{s}\left(\mathfrak{q}^{s-1}\right)} u_{s}(K) \boldsymbol{e}_{s}
$$

with $u_{s}(K)=\prod_{n=1, n \neq s}^{p-1}\left(K-\mathfrak{q}^{s-1-2 n}\right)$, and $\boldsymbol{e}_{s}$ are the central primitive idempotents of $\overline{\mathcal{U}}_{\mathfrak{q}} s \ell(2)$ given in App. $\mathbf{A}$. 
The comultiplication in $\mathcal{L} \mathcal{U}_{\mathfrak{q}} s \ell(2)$ is given in (2.4) for the $E, F$, and $K$ generators and

$$
\begin{aligned}
& \Delta(e)=e \otimes 1+K^{p} \otimes e+\frac{1}{[p-1] !} \sum_{r=1}^{p-1} \frac{\mathfrak{q}^{r(p-r)}}{[r]} K^{p} E^{p-r} \otimes E^{r} K^{-r}, \\
& \Delta(f)=f \otimes 1+K^{p} \otimes f+\frac{(-1)^{p}}{[p-1] !} \sum_{s=1}^{p-1} \frac{\mathfrak{q}^{-s(p-s)}}{[s]} K^{p+s} F^{s} \otimes F^{p-s},
\end{aligned}
$$

an explicit form of $\Delta(h)=\frac{1}{2}[\Delta(e), \Delta(f)]$ is very bulky and we do not give it here.

The antipode $S$ and the counity $\epsilon$ are given in (2.5)-(2.6) and

$$
\begin{gathered}
S(e)=-K^{p} e, \quad S(f)=-K^{p} f, \quad S(h)=-h, \\
\epsilon(e)=\epsilon(f)=\epsilon(h)=0 .
\end{gathered}
$$

2.2.2. $\mathcal{L} \mathcal{U}_{\mathfrak{q}} s \ell(2)$ through divided powers. The quantum group $\mathcal{L} \mathcal{U}_{\mathfrak{q}} s \ell(2)$ can be realized as the Lusztig extension of the restricted quantum group $\bar{U}_{\mathfrak{q}} s \ell(2)$ by divided powers of the $E$ and $F$ generators. In the usual quantum $\mathcal{U}_{\mathfrak{q}} s \ell(2)$ with the relations (2.2), (2.4), (2.5), and (2.6) and with generic $\mathfrak{q}$, the $e, f$ and $h$ exist as the following elements,

$$
e=\frac{1}{[p] !} K^{p} E^{p}, \quad f=\frac{(-1)^{p}}{[p] !} F^{p},
$$

and the Cartan element

$$
\begin{gathered}
h=\frac{1}{2}[e, f]=\frac{1-\mathfrak{q}^{2 p}}{2} e f+\frac{\mathfrak{q}^{2 p}}{2 p\left(\mathfrak{q}-\mathfrak{q}^{-1}\right)}\left(\frac{\mathbf{1}-\left(-\mathfrak{q}^{p}\right)^{p-1} K^{2 p}}{[p]}+\sum_{r=1}^{p-1} \frac{(-1)^{r} \mathfrak{q}^{r(p-1)}[p-1] !}{[p-r] ![r] !} K^{2 r}\right)+ \\
+\frac{(-1)^{p+1}}{2[p-1] !} K^{p} \sum_{n=1}^{p-1} \frac{(-1)^{n}[p-1] !}{([p-n] !)^{2}[n] !} \prod_{k=0}^{p-n-1} \frac{C-\mathfrak{q}^{-(2 k+1)} K-\mathfrak{q}^{(2 k+1)} K^{-1}}{\left(\mathfrak{q}-\mathfrak{q}^{-1}\right)^{2}} \prod_{r=1}^{n} \frac{\mathfrak{q}^{r-1} K-\mathfrak{q}^{-r+1} K^{-1}}{\mathfrak{q}-\mathfrak{q}^{-1}},
\end{gathered}
$$

where the $\mathcal{U}_{\mathfrak{q}} s \ell(2)$ Casimir element $\boldsymbol{C}$ is given in App. $\mathbf{A}$. The commutation relations between these elements and their comultiplication, antipode, and counity satisfy (2.7)(2.16) when $\mathfrak{q} \rightarrow e^{i \pi / p}$.

\section{REPRESENTATIONS OF $\mathcal{L} \mathcal{U}_{\mathfrak{q}} s \ell(2)$}

To describe the category $\mathcal{C}_{p}$ of finite-dimensional $\mathcal{L} \mathcal{U}_{\mathfrak{q}} s \ell(2)$-modules, we first study irreducible $\mathcal{L} \mathcal{U}_{\mathfrak{q}} s \ell(2)$-modules in 3.1 and then obtain essential information about possible extensions between them in $\mathbf{3 . 2}$. This let us construct all finite-dimensional projective $\mathcal{L} \mathcal{U}_{\mathfrak{q}} s \ell(2)$-modules in 3.3 . Then in $\mathbf{3 . 4}$, we decompose the representation category $\mathcal{C}_{p}$ into a direct sum of two full subcategories $\mathcal{C}_{p}^{+}$and $\mathcal{C}_{p}^{-}$. The subcategory $\mathcal{C}_{p}^{+}$is then identified with a tensor category of the Virasoro algebra representations. 
3.1. Irreducible $\mathcal{L} \mathcal{U}_{\mathfrak{q}} s \ell(2)$-modules. An irreducible $\mathcal{L} \mathcal{U}_{\mathfrak{q}} s \ell(2)$-module $X_{s, r}^{ \pm}$is labeled by $( \pm, s, r)$, with $1 \leqslant s \leqslant p$ and $r \in \mathbb{N}$, and has the highest weights $\pm \mathfrak{q}^{s-1}$ and $\frac{r-1}{2}$ with respect to $K$ and $h$ generators, respectively. The $s r$-dimensional module $X_{s, r}^{ \pm}$is spanned by elements $\mathrm{a}_{n, m}^{ \pm}, 0 \leqslant n \leqslant s-1,0 \leqslant m \leqslant r-1$, where $\mathrm{a}_{0,0}^{ \pm}$is the highest-weight vector and the left action of the algebra on $X_{s, r}^{ \pm}$is given by

$$
\begin{aligned}
K \mathrm{a}_{n, m}^{ \pm} & = \pm \mathfrak{q}^{s-1-2 n} \mathrm{a}_{n, m}^{ \pm}, & & h \mathrm{a}_{n, m}^{ \pm}=\frac{1}{2}(r-1-2 m) \mathrm{a}_{n, m}^{ \pm}, \\
E \mathrm{a}_{n, m}^{ \pm} & = \pm[n][s-n] \mathrm{a}_{n-1, m}^{ \pm}, & & e \mathrm{a}_{n, m}^{ \pm}=m(r-m) \mathrm{a}_{n, m-1}^{ \pm}, \\
F \mathrm{a}_{n, m}^{ \pm} & =\mathrm{a}_{n+1, m}^{ \pm}, & & f \mathrm{a}_{n, m}^{ \pm}=\mathrm{a}_{n, m+1}^{ \pm},
\end{aligned}
$$

where we set $\mathrm{a}_{-1, m}^{ \pm}=\mathrm{a}_{n,-1}^{ \pm}=\mathrm{a}_{s, m}^{ \pm}=\mathrm{a}_{n, r}^{ \pm}=0$.

3.1.1. Remark. The element $A$ defined in (2.12) and (2.11) is represented in an irreducible representation of $\mathcal{L} \mathcal{U}_{\mathfrak{q}} s \ell(2)$ by an operator acting as the identity on the highest-weight vector and zero on all other vectors. Therefore, as it follows from the relations (2.8)(2.11), the $E, F$, and $K$ generators of the subalgebra $\overline{\mathcal{U}}_{\mathfrak{q}} s \ell(2)$ commute on $X_{s, r}^{ \pm}$with the $e, f$, and $h$ generators of the subalgebra $s \ell(2)$.

3.2. Extensions among irreducibles. Here, we study possible extensions between irreducible $\mathcal{L} \mathcal{U}_{\mathfrak{q}} s \ell(2)$-modules to construct indecomposable modules in what follows. Let $A$ and $C$ be left $\mathcal{L} \mathcal{U}_{\mathfrak{q}} s \ell(2)$-modules. We say that a short exact sequence of $\mathcal{L} \mathcal{U}_{\mathfrak{q}} s \ell(2)-$ modules $0 \rightarrow A \rightarrow B \rightarrow C \rightarrow 0$ is an extension of $C$ by $A$, and we let $\operatorname{Ext}_{\frac{\mathcal{L} \mathcal{U}_{q}}{1}}(C, A)$ denote the set of equivalence classes (see, e.g., [32]) of extensions of $C$ by $A$.

3.2.1. Lemma. For $1 \leqslant s \leqslant p-1, r \in \mathbb{N}$ and $\alpha, \alpha^{\prime} \in\{+,-\}$, there are vector-space isomorphisms

$$
\operatorname{Ext}_{\overline{\mathcal{L u}}_{q}}^{1}\left(X_{s, r}^{\alpha}, X_{s^{\prime}, r^{\prime}}^{\alpha^{\prime}}\right) \cong \begin{cases}\mathbb{C}, & \alpha^{\prime}=-\alpha, s^{\prime}=p-s, r^{\prime}=r \pm 1, \\ 0, & \text { otherwise. }\end{cases}
$$

There are no nontrivial extensions between $X_{p, r}^{ \pm}$and any irreducible module.

Proof. We first recall [20] that the space $\operatorname{Ext}_{\bar{u}_{q}}^{1}$ of extensions between irreducible modules over the subalgebra $\overline{\mathcal{U}}_{\mathfrak{q}} s \ell(2)$ is at most two-dimensional and there exists a nontrivial extension only between $X_{s}^{ \pm}$and $X_{p-s}^{\mp}$, where $1 \leqslant s \leqslant p-1$ and we set $X_{s}^{ \pm}=\left.X_{s, 1}^{ \pm}\right|_{\bar{u}_{\mathfrak{q}} s \ell(2)}$. Moreover, there is an action of $\mathcal{L} \mathcal{U}_{\mathfrak{q}} s \ell(2)$ on projective resolutions for irreducible $\overline{\mathcal{U}}_{\mathfrak{q}} s \ell(2)$ modules and this generates an action of the quotient-algebra $s \ell(2)$ on the corresponding cochain complexes and their cohomologies. Therefore, for an irreducible $\mathcal{X}$ and an $\overline{\mathcal{U}}_{\mathfrak{q}} s \ell(2)$-module $\mathcal{M}$, all extension groups $\operatorname{Ext}_{\overline{\mathcal{U}}_{q}}^{\bullet}(\mathcal{X}, \mathcal{M})$ are $s \ell(2)$-modules. In particular, the space $\operatorname{Ext}_{\bar{u}_{q}}^{1}\left(X_{s}^{ \pm}, X_{p-s}^{\mp}\right)$ is the $s \ell(2)$-doublet and there is a nontrivial $s \ell(2)$ action on all Hochschild cohomologies of $\overline{\mathcal{U}}_{\mathfrak{q}} s \ell(2)$ (see [31]). 
Next, to calculate the first extension groups between the irreducible $\mathcal{L} \mathcal{U}_{\mathfrak{q}} s \ell(2)$-modules, we use the Serre-Hochschild spectral sequence with respect to the subalgebra $\overline{\mathcal{U}}_{\mathfrak{q}} s \ell(2)$ and the quotient-algebra $s \ell(2)$. The spectral sequence is degenerate at the second term due to the semisimplicity of the quotient algebra and we thus obtain

$$
\operatorname{Ext}_{\overline{\mathcal{L U}}_{q}}^{1}\left(X_{s, r}^{\alpha}, X_{s^{\prime}, r^{\prime}}^{\alpha^{\prime}}\right)=H^{0}\left(s \ell(2), \operatorname{Ext}_{\overline{\mathcal{u}}_{q}}^{1}\left(X_{s, r}^{\alpha}, X_{s^{\prime}, r^{\prime}}^{\alpha^{\prime}}\right)\right)
$$

where the right-hand side is the vector space of the $s \ell(2)$-invariants in the $s \ell(2)$-module $\operatorname{Ext}_{\bar{u}_{q}}^{1}\left(X_{s, r}^{\alpha}, X_{s^{\prime}, r^{\prime}}^{\alpha^{\prime}}\right)$. This module is nonzero only in the case $\alpha^{\prime}=-\alpha, s^{\prime}=p-s$ and isomorphic to the tensor product $X_{2} \otimes X_{r} \otimes X_{r^{\prime}}$ of the $s \ell(2)$ modules, where $X_{r}$ is the $r$-dimensional module. Obviously, the tensor product contains a trivial $s \ell(2)$-module only in the case $r^{\prime}=r \pm 1$. This completes the proof.

3.2.2. Example. As an example, we describe an extension of $X_{s, r}^{ \pm}$by $X_{p-s, r+1}^{\mp}$. This can be realized as an extension of $r$ copies $X_{s}^{ \pm}$by $(r+1)$ copies $X_{p-s}^{\mp}$ of the irreducible modules over the subalgebra $\overline{\mathcal{U}}_{\mathfrak{q}} s \ell(2)$,

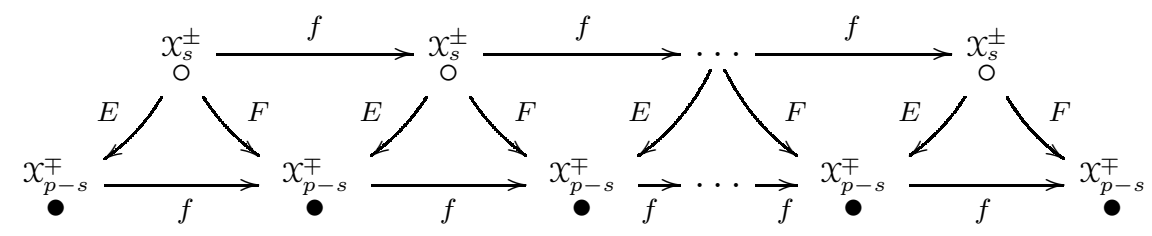

with the indicated action of the $E$ and $F$ generators mixing $X_{s}^{ \pm}$with $X_{p-s}^{\mp}$ modules, and with the $e$ and $f$ generators mapping different copies with the same sign. The extension thus constructed can be depicted as

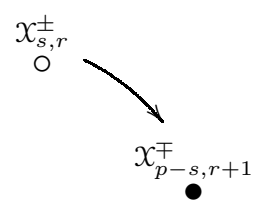

with the convention that the arrow is directed to the submodule in the bottom marked by $\bullet$, in contrast to the subquotient $\circ$ in the top.

3.3. Projective $\mathcal{L} \mathcal{U}_{\mathfrak{q}} s \ell(2)$-modules. We next construct projective $\mathcal{L} \mathcal{U}_{\mathfrak{q}} s \ell(2)$-modules as projective covers of irreducible modules. A projective cover of an irreducible module is a "maximal" indecomposable module that can be mapped onto the irreducible. Lem. 3.2.1 state that we can "glue" two irreducible modules into an indecomposable module only in the case if the irreducibles have opposite signs of the $\alpha$-index, the difference between the two $r$-indexes equals to one and the sum of the two $s$-indexes is equal to $p$. Therefore, to construct a projective cover for $\chi_{s, r}^{ \pm}, 1 \leqslant s \leqslant p-1$ and $r \geqslant 2$, we first have to obtain a nontrivial extension of $X_{s, r}^{ \pm}$by the maximal number of irreducible modules, that is, by

$$
X_{p-s, r-1}^{\mp} \otimes \operatorname{Ext}_{\overline{\mathcal{L}}_{q}}^{1}\left(X_{s, r}^{ \pm}, X_{p-s, r-1}^{\mp}\right) \oplus X_{p-s, r+1}^{\mp} \otimes \operatorname{Ext}_{{\bar{L} \mathcal{U}_{q}}^{1}}\left(X_{s, r}^{ \pm}, X_{p-s, r+1}^{\mp}\right),
$$


which is

$$
0 \rightarrow X_{p-s, r-1}^{\mp} \oplus X_{p-s, r+1}^{\mp} \rightarrow \mathcal{M}_{s, r}^{ \pm} \rightarrow X_{s, r}^{ \pm} \rightarrow 0 .
$$

where $\mathcal{M}_{s, r}^{ \pm}$is an indecomposable module. Next, to find the projective cover of $X_{s, r}^{ \pm}$, we extend the submodule $X_{p-s, r-1}^{\mp} \oplus X_{p-s, r+1}^{\mp} \subset \mathcal{M}_{s, r}^{ \pm}$by the maximal number of irreducible modules, that is, by $X_{s, r-2}^{ \pm} \oplus 2 X_{s, r}^{ \pm} \oplus X_{s, r+2}^{ \pm}$. The compatibility with the $\mathcal{L} \mathcal{U}_{\mathfrak{q}} s \ell(2)$-algebra relations (with $F^{p}=E^{p}=0$ and (2.11) in particular) leads to an extension corresponding to the module $\mathcal{P}_{s, r}^{ \pm}$with the following subquotient structure:

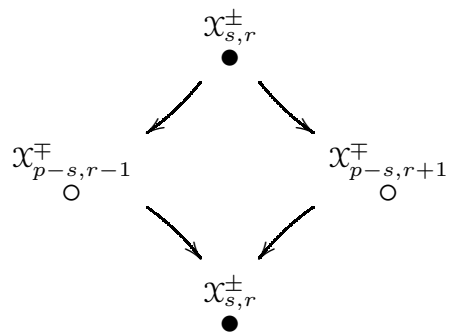

and the $\mathcal{L} \mathcal{U}_{\mathfrak{q}} s \ell(2)$ action is explicitly described in App. B. This module being restricted to the subalgebra $\bar{U}_{\mathfrak{q}} s \ell(2)$ is a direct sum of projective modules that covers the direct sum $\oplus_{i=1}^{r} X_{s}^{ \pm}$, where we set $X_{s}^{ \pm}=\left.X_{s, 1}^{ \pm}\right|_{\bar{u}_{\mathfrak{q}} s \ell(2)}$. Therefore, the $\mathcal{P}_{s, r}^{ \pm}$module is the projective cover of $X_{s, r}^{ \pm}$, for $1 \leqslant s \leqslant p-1$ and $r \geqslant 2$. Similar procedure gives the projective cover $\mathcal{P}_{s, 1}^{ \pm}$for the irreducible module $\mathcal{X}_{s, 1}^{ \pm}$with the following subquotient structure:

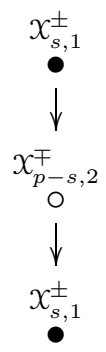

and the $\mathcal{L} \mathcal{U}_{\mathfrak{q}} s \ell(2)$ action is also explicitly described in App. B.

A "half" of these projective modules is then identified in the fusion algebra calculated below in Sec. 4 with some logarithmic Virasoro representations.

3.3.1. Remark. We note there are no additional parameters distinguishing nonisomorphic indecomposable $\mathcal{L} \mathcal{U}_{\mathfrak{q}} s \ell(2)$-modules with the same subquotient structure as in (3.4) and (3.5). This trivially follows from the fact that all infinitesimal deformations of homomorphisms $f: \mathcal{L} \mathcal{U}_{\mathfrak{q}} s \ell(2) \rightarrow \operatorname{End}\left(\mathcal{P}_{s, r}^{ \pm}\right)$continuing infinitesimal deformations of the algebra $\operatorname{End}\left(\mathcal{P}_{s, r}^{ \pm}\right)$are in one-to-one correspondence with elements in the cohomology space $H^{1}\left(\mathcal{L} \mathcal{U}_{\mathfrak{q}} s \ell(2), \operatorname{End}\left(\mathcal{P}_{s, r}^{ \pm}\right)\right)$. Since the module $\operatorname{End}\left(\mathcal{P}_{s, r}^{ \pm}\right)$is a direct sum of projective modules then the cohomologies space $H^{1}$ is trivial. 
3.3.2. Semisimple length of a module. Let $\mathcal{N}$ be a $\mathcal{L} \mathcal{U}_{\mathfrak{q}} s \ell(2)$-module. We define a semisimple filtration of $\mathcal{N}$ as a tower of submodules

$$
\mathcal{N}=\mathcal{N}_{0} \supset \mathcal{N}_{1} \supset \ldots \supset \mathcal{N}_{l}=0
$$

such that each quotient $\mathcal{N}_{i} / \mathcal{N}_{i+1}$ is semisimple. The number $l$ is called the length of the filtration. In the set of semisimple filtrations of $\mathcal{N}$, there exists a filtration with the minimum length $\ell$. We call $\ell$ the semisimple length of $\mathcal{N}$.

Evidently, an indecomposable module has the semisimple length not less than 2. Any semisimple module has the semisimple length 1 .

\subsubsection{Proposition.}

(1) Every indecomposable $\mathcal{L} \mathcal{U}_{\mathfrak{q}} s \ell(2)$-module with the semisimple length 3 is isomorphic to $\mathcal{P}_{s, r}^{ \pm}$, for some $s \in\{1,2, \ldots, p-1\}$ and some finite $r \in \mathbb{N}$.

(2) There are no indecomposable modules with the semisimple length greater than 3.

Proof. Consider a module $\mathcal{M}$ with a nonvanishing mapping $\mathcal{P}_{s, r}^{ \pm} \rightarrow \mathcal{M}$ that covers $\mathcal{X}_{s, r}^{ \pm}$. In the case when the mapping is an embedding, we note that the projective module $\mathcal{P}_{s, r}^{ \pm}$is also an injective module (the contragredient one to a projective module) and is therefore a direct summand in any module into which it is embedded. In the case with a nonvanishing kernel of the mapping, the kernel contains the submodule $\mathcal{X}_{s, r}^{ \pm}$of $\mathcal{P}_{s, r}^{ \pm}$. Therefore, the subquotient actually belongs to a direct summand in $\mathcal{M}$ with the semisimple length 2 or 1 .

We thus conclude that there are no indecomposable modules with the semisimple length 4 ("higher" than $\mathcal{P}_{s, r}^{ \pm}$) and every $\mathcal{L} \mathcal{U}_{\mathfrak{q}} s \ell(2)$-module with the semisimple length 3 is isomorphic to a direct sum of $\mathcal{P}_{s, r}^{ \pm}$.

3.4. Decomposition of the category $\mathcal{C}_{p}$. Here, we describe the category $\mathcal{C}_{p}$ of finitedimensional $\mathbb{Z}^{2}$-graded modules over $\mathcal{L} \mathcal{U}_{\mathfrak{q}} s \ell(2)$. We use the results about possible extensions between irreducible modules over $\mathcal{L} \mathcal{U}_{\mathfrak{q}} s \ell(2)$, Lem. 3.2.1, to state the following decomposition theorem.

\subsubsection{Theorem.}

(1) The category $\mathrm{C}_{p}$ of finite-dimensional $\mathcal{L} \mathcal{U}_{\mathfrak{q}} s \ell(2)$-modules has the decomposition

$$
\mathcal{C}_{p}=\bigoplus_{s=1}^{p-1} \mathcal{C}^{+}(s) \oplus \mathcal{C}^{-}(s) \oplus \bigoplus_{r \in \mathbb{N}} \mathcal{S}^{+}(r) \oplus \mathcal{S}^{-}(r),
$$

where each direct summand is a full indecomposable subcategory.

(2) Each of the full subcategories $\mathcal{S}^{+}(r)$ and $\mathcal{S}^{-}(r)$ is semisimple and contains precisely one irreducible module, $X_{p, r}^{+}$and $X_{p, r}^{-}$respectively.

(3) Each $\mathrm{C}^{+}(s)$ contains the family of irreducible modules $X_{s, 2 r-1}^{+}$and $X_{p-s, 2 r}^{-}, r \in \mathbb{N}$.

(4) Each $\mathrm{C}^{-}(s)$ contains the family of irreducible modules $X_{s, 2 r}^{+}$and $X_{p-s, 2 r-1}^{-}, r \in \mathbb{N}$. 
We recall [20] the decomposition of the representation category $\bar{\complement}_{p}$ for $\overline{\mathcal{U}}_{\mathfrak{q}} s \ell(2)$ :

$$
\bar{\complement}_{p}=\bigoplus_{s=0}^{p} \bar{\complement}(s)
$$

where each $\overline{\mathrm{C}}(s)$ is a full indecomposable subcategory and contains two irreducibles $X_{s}^{+}$ and $X_{p-s}^{-}$, for $1 \leqslant s \leqslant p-1$, that can be composed into an indecomposable module. The subcategories $\overline{\mathrm{C}}(0)$ and $\overline{\mathrm{C}}(p)$ are semisimple and contain $\mathcal{X}_{p}^{+}$and $\mathcal{X}_{p}^{-}$respectively.

3.4.2. Lemma. An indecomposable $\mathcal{L} \mathcal{U}_{\mathfrak{q}} s \ell(2)$-module considered a $\overline{\mathcal{U}}_{\mathfrak{q}} s \ell(2)$-module is a direct sum of indecomposable modules from the full subcategory $\overline{\mathcal{C}}(s)$ in (3.6) for some fixed $s \in\{1,2, \ldots, p-1\}$.

In the following section, we show that the full subcategory

$$
\mathrm{C}_{p}^{+}=\bigoplus_{s=1}^{p-1} \mathcal{C}^{+}(s) \oplus \bigoplus_{\text {odd } r \geqslant 1} \mathcal{S}^{+}(r) \oplus \bigoplus_{\text {even } r \geqslant 2} \mathcal{S}^{-}(r)
$$

in the category $\mathcal{C}_{p}=\mathcal{C}_{p}^{+} \oplus \mathcal{C}_{p}^{-}$is closed under the tensor product operation and is identified with a tensor category of representations for the Virasoro algebra $\mathcal{V}_{p}$. This gives fusion rules for logarithmic $(1, p)$ models with the chiral symmetry $\mathcal{V}_{p}$.

\section{The FUSION ALGEBRA}

In this section, we calculate tensor products between $\mathcal{L} \mathcal{U}_{\mathfrak{q}} s \ell(2)$ irreducible and projective modules introduced in Sec. 3. To decompose the tensor products, we first show how to extend results for $\mathcal{L} \mathcal{U}_{\mathfrak{q}} s \ell(2)$-modules with the $s \ell(2)$-index $r=1$ to modules with $r>1$.

4.1. Lemma. For $1 \leqslant s \leqslant p$ and $r \in \mathbb{N}$, we have isomorphisms of $\mathcal{L} \mathcal{U}_{\mathfrak{q}} s \ell(2)$-modules,

$$
X_{s, r}^{+} \cong X_{1, r}^{ \pm} \otimes X_{s, 1}^{ \pm} \cong X_{s, 1}^{ \pm} \otimes X_{1, r}^{ \pm}, \quad X_{s, r}^{-} \cong X_{1, r}^{ \pm} \otimes X_{s, 1}^{\mp} \cong X_{s, 1}^{ \pm} \otimes X_{1, r}^{\mp}
$$

and

$$
\mathcal{P}_{s, r}^{+} \cong X_{1, r}^{ \pm} \otimes \mathcal{P}_{s, 1}^{ \pm} \cong \mathcal{P}_{s, 1}^{ \pm} \otimes X_{1, r}^{ \pm}, \quad \mathcal{P}_{s, r}^{-} \cong X_{1, r}^{ \pm} \otimes \mathcal{P}_{s, 1}^{\mp} \cong \mathcal{P}_{s, 1}^{ \pm} \otimes X_{1, r}^{\mp}
$$

Proof. As an example, we consider $X_{1, r}^{+} \otimes \mathcal{P}_{s, 1}^{+}$. In this case, the comultiplication takes the following form:

$$
\begin{aligned}
& \Delta(E)=\mathbf{1} \otimes E, \quad \Delta(F)=K^{-1} \otimes F, \quad \Delta(K)=K \otimes K \\
& \Delta(e)=K^{p} \otimes e+e \otimes 1, \quad \Delta(f)=K^{p} \otimes f+f \otimes 1, \quad \Delta(h)=1 \otimes h+h \otimes 1 .
\end{aligned}
$$

Let $\mathrm{a}_{m}$, with $0 \leqslant m \leqslant r-1$, denotes the basis in (3.1) - (3.3) for $X_{1, r}^{+}$and $\mathrm{t}_{n}, \mathrm{~b}_{n}, \mathrm{r}_{k, 0}$, $\mathrm{r}_{k, 1}$, with $0 \leqslant n \leqslant s-1$ and $0 \leqslant k \leqslant p-s-1$, is the basis in App $\underline{\mathbf{B}}$ for $\mathcal{P}_{s, 1}^{ \pm}$. Then, the basis for the product is

$$
\mathrm{t}_{n, m}=\mathrm{a}_{m} \otimes \mathrm{t}_{n}, \quad \mathrm{~b}_{n, m}=\mathrm{a}_{m} \otimes \mathrm{b}_{n},
$$




$$
\mathrm{r}_{k, i}=\mathrm{a}_{i} \otimes \mathrm{r}_{k, 0}+i \mathrm{a}_{i-1} \otimes \mathrm{r}_{k, 1}, \quad \mathrm{l}_{k, j}=\mathrm{a}_{j} \otimes \mathrm{r}_{k, 0}-(r-j) \mathrm{a}_{j-1} \otimes \mathrm{r}_{k, 1},
$$

where $0 \leqslant i \leqslant r, 1 \leqslant j \leqslant r-1$ and we assume $\mathrm{a}_{r} \equiv 0$. The action of all the generators coincides with the one in $\mathcal{P}_{s, r}^{+}$explicitly described in App. B. The other cases have a similar proof.

Then, we calculate tensor products starting with simplest cases. Tensor products of modules with arbitrary $r$-indexes are based on their $r=1$ cases and tensor products of a projective module with an irreducible or a projective module are based on tensor products of their irreducible subquotients and submodules.

4.2. Fusion of irreducible modules. The case of tensor products of two irreducibles with the $s \ell(2)$-index $r=1$ is the simplest one.

4.2.1. Lemma. For $1 \leqslant s_{1}, s_{2} \leqslant p-1$, we have

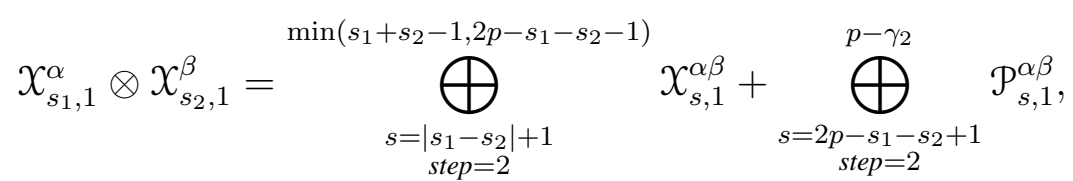

where $\gamma_{2}=\left(s_{1}+s_{2}+p+1\right) \bmod 2$.

Proof. The tensor product restricted to the subalgebra $\overline{\mathcal{U}}_{\mathfrak{q}} s \ell(2)$ has the well known decomposition [19, 21] (see also [33] for more general case of Taft Hopf algebras). In this decomposition, each two direct summands belongs to pairwise different indecomposable subcategories in the category $\bar{\complement}_{p}$ (see (3.6) ) and according to Lem. 3.4.2 any direct sum of them can not be combined into an indecomposable $\mathcal{L} \mathcal{U}_{\mathfrak{q}} s \ell(2)$-module. Therefore, the action of the $e, f$, and $h$ generators is unambiguously defined.

Using Lem. 4.1, we can easily extend this result to arbitrary $r$-index.

4.2.2. Theorem. For $1 \leqslant s_{1} \leqslant p-1, r_{1}, r_{2} \in \mathbb{N}$, we have

$$
X_{s_{1}, r_{1}}^{\alpha} \otimes X_{s_{2}, r_{2}}^{\beta}=\bigoplus_{\substack{r=\left|r_{1}-r_{2}\right|+1 \\ \text { step }=2}}^{r_{1}+r_{2}-1}\left(\bigoplus_{\substack{s=\left|s_{1}-s_{2}\right|+1 \\ \text { step }=2}}^{\min \left(s_{1}+s_{2}-1,2 p-s_{1}-s_{2}-1\right)} X_{s, r}^{\alpha \beta}+\bigoplus_{\substack{s=2 p-s_{1}-s_{2}+1 \\ \text { step }=2}}^{p-\gamma_{2}} \mathcal{P}_{s, r}^{\alpha \beta}\right)
$$

where $\gamma_{2}=\left(s_{1}+s_{2}+p+1\right) \bmod 2$.

Proof. We only need to show that $X_{1, r_{1}}^{+} \otimes X_{1, r_{2}}^{+}=\bigoplus_{\substack{r=\left|r_{1}-r_{2}\right|+1 \\ \text { step=2 }}}^{r_{1}+r_{2}-1} X_{1, r}^{+}$. This trivially follows from the comultiplication restricted on this tensor product,

$$
\begin{aligned}
& \Delta(E)=0, \quad \Delta(F)=0, \quad \Delta(K)=1 \otimes 1, \\
& \Delta(e)=1 \otimes e+e \otimes 1, \quad \Delta(f)=1 \otimes f+f \otimes 1, \quad \Delta(h)=1 \otimes h+h \otimes 1,
\end{aligned}
$$

which coincides with the usual comultiplication for the $s \ell(2)$. 
4.3. Fusion with projectives. The above results allow us to decompose tensor products of irreducible modules with projective ones and of two projective modules. We note that in both cases the tensor product must contain only projectives.

To decompose the tensor product of an irreducible module and a projective one, we start with the $r=1$ case as well. We consider irreducible subquotients and submodules of the projective module and calculate their tensor products with the irreducible module. Projectives obtained from these tensor products are direct summands because any projective $\mathcal{L} \mathcal{U}_{\mathfrak{q}} s \ell(2)$-module is also injective (the contragredient one to a projective module) and is therefore a direct summand in any module into which it is embedded. Irreducibles obtained from the tensor products are subquotients of projective modules in the whole tensor product. This procedure thus gives a decomposition of the tensor product of an irreducible module with a projective module.

4.3.1. Lemma. For $1 \leqslant s_{1} \leqslant p-1$, we have

$$
X_{s_{1}, 1}^{\alpha} \otimes \mathcal{P}_{s_{2}, 1}^{\beta}=\bigoplus_{\substack{s=\left|s_{1}-s_{2}\right|+1 \\ \text { step }=2}}^{\substack{\min \left(s_{1}+s_{2}-1, 2 p-s_{1}-s_{2}-1\right)}} \mathcal{P}_{s, 1}^{\alpha \beta}+2 \bigoplus_{\substack{s=2 p-s_{1}-s_{2}+1 \\ \text { step }=2}}^{p-\gamma_{2}} \mathcal{P}_{s, 1}^{\alpha \beta}+\bigoplus_{\substack{s=p-s_{1}+s_{2}+1 \\ \text { step }=2}}^{p-\gamma_{1}} \mathcal{P}_{s, 2}^{-\alpha \beta},
$$

where $\gamma_{1}=\left(s_{1}+s_{2}+1\right) \bmod 2$, and $\gamma_{2}=\left(s_{1}+s_{2}+p+1\right) \bmod 2$.

Proof. The projective module $\mathcal{P}_{s_{2}, 1}^{\beta}$ contains three irreducible subquotients. Let denote them according to their position in the diagram (3.5), for simplicity. The top $X_{s_{2}, 1}^{\beta}$ is marked as $T$, the bottom as $B$, and the middle $X_{p-s_{2}, 2}^{-\beta}$ as $M$. The tensor product of the irreducible module $X_{s_{1}, 1}^{\alpha}$ with each of them contains irreducible terms which can uniquely combain into a projective module. These projectives are the first sum in the right-hand side of (4.1). The tensor product $X_{s_{1}, 1}^{\alpha} \otimes M$ for $s_{1}>s_{2}$ gives the $\bigoplus_{s=p-s_{1}+s_{2}+1}^{p-\gamma_{1}} \mathcal{P}_{s, 2}^{-\alpha \beta}$ terms in (4.1). The tensor products $X_{s_{1}, 1}^{\alpha} \otimes T$ and $X_{s_{1}, 1}^{\alpha} \otimes B$ give $\bigoplus_{s=2 p-s_{1}-s_{2}+1}^{p-\gamma_{2}} \mathcal{P}_{s, 1}^{\alpha \beta}$ terms in (4.1), when $s_{1}>p-s_{2}$.

We also decompose tensor products of two projective modules for the $r=1$ case analogously as in Lem. 4.3.1.

$$
\begin{aligned}
& \begin{array}{lcl}
\min \left(s_{1}+s_{2}-1,\right. & \min \left(p-s_{1}+s_{2}-1,\right. \\
\left.2 p-s_{1}-s_{2}-1\right) & \left.p+s_{1}-s_{2}-1\right)
\end{array} \quad p-\gamma_{1} \\
& \mathcal{P}_{s_{1}, 1}^{\alpha} \otimes \mathcal{P}_{s_{2}, 1}^{\beta}=2 \bigoplus_{\substack{s=\left|s_{1}-s_{2}\right|+1 \\
\text { step }=2}} \mathcal{P}_{s, 1}^{\alpha \beta}+\bigoplus_{\substack{s=\left|p-s_{1}-s_{2}\right|+1 \\
\text { step }=2}}^{p} \mathcal{P}_{s, 2}^{-\alpha \beta}+2 \bigoplus_{\substack{s=\min \left(p-s_{1}+s_{2}+1, p+s_{1}-s_{2}+1\right), \text { step }=2}}^{p-\gamma_{1}} \mathcal{P}_{s, 2}^{-\alpha \beta} \\
& +4 \bigoplus_{\substack{s=2 p-s_{1}-s_{2}+1 \\
\text { step }=2}}^{p-\gamma_{2}} \mathcal{P}_{s, 1}^{\alpha \beta}+\bigoplus_{\substack{s=s_{1}+s_{2}+1 \\
\text { step }=2}}^{p-\gamma_{2}}\left(\mathcal{P}_{s, 1}^{\alpha \beta}+\mathcal{P}_{s, 3}^{\alpha \beta}\right)
\end{aligned}
$$

Using Lem. 4.1, we now extend these results for arbitrary $r$-index and obtain the final results in Thm.1.1. 
4.4. Relations to Virasoro fusion algebra. In (1.1), we identify $\mathcal{L} \mathcal{U}_{\mathfrak{q}} s \ell(2)$ irreducible and projective modules with irreducible and logarithmic modules of the Virasoro algebra $\mathcal{V}_{p}$. Under the identification, the tensor products of $\mathcal{L} \mathcal{U}_{\mathfrak{q}} s \ell(2)$-modules coincide with the fusion of the corresponding [GaberdielKausch]-modules. In other words, there exists a tensor functor from the category $\mathrm{C}_{p}^{+}$to the category of $\mathcal{V}_{p}$-modules with dimension of $L_{0}$ Jordan cells not greater than 2 . The functor establishes a one to one correspondence between simple objects of two categories but is not an equivalence because the Virasoro category contains more morphisms between simple objects and more indecomposable objects than $\mathcal{C}_{p}^{+}$. In particular, Virasoro Verma modules have no counterpart on the quantum group side. $\mathcal{V}_{p}$ also admits a class of modules with two dimensional $L_{0}$ Jordan cells enumerated by a projective parameter. All these modules have the same subquotient structure (3.4) nevertheless are parawise different and only module with a special value of the parameter has a counterpart on the quantum group side (see Rem. 3.3.1). The details of the correspondence between $\mathcal{V}_{p}$ and $\mathcal{L} \mathcal{U}_{\mathfrak{q}} s \ell(2)$ indecomposable modules will be written in the future paper [34].

\section{Conclusions}

In the paper, we identified the fusion [25] of the $\mathcal{L} \mathcal{M}(1, p)$ logarithmic models with the tensor products of $\mathcal{L} \mathcal{U}_{\mathfrak{q}} s \ell(2)$ irreducible and projective modules. The Virasoro chiral algebra $\mathcal{V}_{p}$ of $\mathcal{L} \mathcal{M}(1, p)$ and $\mathcal{L} \mathcal{U}_{\mathfrak{q}} s \ell(2)$ centralizes each other in the free-field space of states. This suggests that $\mathcal{V}_{p}$ and $\mathcal{L} \mathcal{U}_{\mathfrak{q}} s \ell(2)$ should be in the Kazhdan-Lusztig duality. However, this duality is more subtle than duality between $\mathcal{W}$ and $\overline{\mathcal{U}}_{\mathfrak{q}} s \ell(2)$ in [19]. In the $\mathcal{V}_{p}-\mathcal{L} \mathcal{U}_{\mathfrak{q}} s \ell(2)$ case there is no equivalence between category $\mathrm{C}_{p}^{+}$and naive category of $\mathcal{V}_{p}$ representations. An identification of a relevant $\mathcal{V}_{p}$ category is an important future problem.

The quantum group $\mathcal{L} \mathcal{U}_{\mathfrak{q}} s \ell(2)$ is the maximal centralizer of the Virasoro algebra $\mathcal{V}_{p}$ on the full chiral space of states. The two commuting actions on the space of states are combined into a bimodule over the Virasoro algebra and the quantum group. This bimodule is expected to be closely related to the regular bimodule for the corresponding quantum group, which will be explicitly constructed in our future paper. The bimodule is resemble the limiting bimodule [2] that corresponds to the vacuum sector in the XXZ model chiral space of states.

In view of the proposed duality between the Virasoro algebra $\mathcal{V}_{p}$ and the quantum group $\mathcal{L} \mathcal{U}_{\mathfrak{q}} s \ell(2)$, there is a correspondence between representation (sub)categories of the quantum group and the Virasoro algebra. This allows constructing infinite series of indecomposable representations for the Virasoro algebra (with the well-known Feigin-Fuchs modules included) and the Felder resolutions in quantum-group terms.

The fusion algebra for the irreducible and indecomposable Virasoro representations was calculated using the comultiplication in the quantum group $\mathcal{L} \mathcal{U}_{\mathfrak{q}} s \ell(2)$. These results are 
in good correspondence with the recent results of Read and Saleur [2] based on a detailed study of quantum XXZ spin chains with the anisotropy parameter related to the deformation parameter of the quantum group being a primitive root of unity. This opens a possibility to investigate XXZ spin chains in terms of $\mathcal{L} \mathcal{U}_{\mathfrak{q}} s \ell(2)$. In particular, coefficients in front of $z$ in characters of multiplicity spaces calculated in [35] are related to multiplicities of $\mathcal{L} \mathcal{U}_{\mathfrak{q}} s \ell(2)$ projective modules in tensor products of it's irreducibles and conjecturally give characters of Temperley-Lieb algebra modules realized in XXZ spin chains.

Acknowledgments. We are grateful to M. Flohr, P. Pearce, J. Rasmussen, H. Saleur and A.M. Semikhatov for valuable discussions and careful reading of the text. The work of BLF was supported in part by RFBR Grant 08-01-00720, RFBR-CNRS-07-01-92214 and LSS-3472.2008.2. The work of AMG was supported in part by RFBR Grant 07-01-00523, by the Grant LSS-1615.2008.2, and by the "Landau", "Dynasty" and "Science Support" foundations. The work of PVB was supported in part by RFBR Grant 07-01-00523. The work of IYuT was supported in part by LSS-1615.2008.2, the RFBR Grant 08-02-01118 and the "Dynasty" foundation.

\section{ApPendix A. Central idempotents}

We recall the central idempotents in $\bar{U}_{\mathfrak{q}} s \ell(2)$ [19]:

$$
\begin{aligned}
& \boldsymbol{e}_{s}=\frac{1}{\psi_{s}\left(\beta_{s}\right)}\left(\psi_{s}(\boldsymbol{C})-\frac{\psi_{s}^{\prime}\left(\beta_{s}\right)}{\psi_{s}\left(\beta_{s}\right)}\left(\boldsymbol{C}-\beta_{s}\right) \psi_{s}(\boldsymbol{C})\right), \quad 1 \leqslant s \leqslant p-1, \\
& \boldsymbol{e}_{0}=\frac{1}{\psi_{0}\left(\beta_{0}\right)} \psi_{0}(\boldsymbol{C}), \quad \boldsymbol{e}_{p}=\frac{1}{\psi_{p}\left(\beta_{p}\right)} \psi_{p}(\boldsymbol{C}),
\end{aligned}
$$

with the polynomials

$$
\begin{aligned}
& \psi_{s}(x)=\left(x-\beta_{0}\right)\left(x-\beta_{p}\right) \prod_{\substack{j=1 \\
j \neq s}}^{p-1}\left(x-\beta_{j}\right)^{2}, \quad 1 \leqslant s \leqslant p-1, \\
& \psi_{0}(x)=\left(x-\beta_{p}\right) \prod_{j=1}^{p-1}\left(x-\beta_{j}\right)^{2}, \quad \psi_{p}(x)=\left(x-\beta_{0}\right) \prod_{j=1}^{p-1}\left(x-\beta_{j}\right)^{2},
\end{aligned}
$$

where $\beta_{j}=\mathfrak{q}^{j}+\mathfrak{q}^{-j}$, and the Casimir element

$$
\boldsymbol{C}=\left(\mathfrak{q}-\mathfrak{q}^{-1}\right)^{2} E F+\mathfrak{q}^{-1} K+\mathfrak{q} K^{-1}=\left(\mathfrak{q}-\mathfrak{q}^{-1}\right)^{2} F E+\mathfrak{q} K+\mathfrak{q}^{-1} K^{-1} .
$$

Appendix B. Projective $\mathcal{L} \mathcal{U}_{\mathfrak{q}} s \ell(2)$-MOdules

Here, we explicitly describe the $\mathcal{L} \mathcal{U}_{\mathfrak{q}} s \ell(2)$ action in the projective module $\mathcal{P}_{s, r}^{ \pm}$. Let $s$ be an integer $1 \leqslant s \leqslant p-1$ and $r \in \mathbb{N}$.

For $r>1$, the projective module $\mathcal{P}_{s, r}^{ \pm}$has the basis

$$
\left\{\mathrm{t}_{n, m}, \mathrm{~b}_{n, m}\right\}_{\substack{0 \leqslant n \leqslant s-1 \\ 0 \leqslant m \leqslant r-1}} \cup\left\{\mathrm{I}_{k, l}\right\}_{\substack{0 \leqslant k \leqslant p-s-1 \\ 1 \leqslant l \leqslant r-1}} \cup\left\{\mathrm{r}_{k, l}\right\}_{\substack{0 \leqslant k \leqslant p-s-1 \\ 0 \leqslant l \leqslant r}},
$$


where $\left\{\mathrm{t}_{n, m}\right\}_{\substack{0 \leqslant n \leqslant s-1 \\ 0 \leqslant m \leqslant r-1}}$ is the basis corresponding to the top module in (3.4), $\left\{\mathrm{b}_{n, m}\right\}_{\substack{0 \leqslant n \leqslant s-1 \\ 0 \leqslant m \leqslant r-1}}$ to the bottom, $\left\{\mathrm{I}_{k, l}\right\}_{\substack{0 \leqslant k \leqslant p-s-1 \\ 1 \leqslant l \leqslant r-1}}$ to the left, and $\left\{\mathrm{r}_{k}\right\}_{\substack{0 \leqslant k \leqslant p-s-1 \\ 0 \leqslant l \leqslant r}}$ to the right module.

For $r=1$, the basis does not contain $\left\{\mathrm{I}_{k, l}\right\}_{\substack{0 \leqslant k \leqslant p-s-1 \\ 1 \leqslant l \leqslant r-1}}$ terms and we imply $\mathrm{I}_{k, l} \equiv 0$ in the action. The $\mathcal{L} \mathcal{U}_{\mathfrak{q}} s \ell(2)$-action on $\mathcal{P}_{s, r}^{ \pm}$is given by

$$
\begin{aligned}
& K \mathrm{t}_{n, m}= \pm \mathfrak{q}^{s-1-2 n} \mathrm{t}_{n, m}, \quad 0 \leqslant n \leqslant s-1, \quad 0 \leqslant m \leqslant r-1, \\
& \left.K\right|_{k, m}=\left.\mp \mathfrak{q}^{p-s-1-2 k}\right|_{k, m}, \quad 0 \leqslant k \leqslant p-s-1, \quad 1 \leqslant m \leqslant r-1, \\
& K r_{k, m}=\mp \mathfrak{q}^{p-s-1-2 k} \boldsymbol{r}_{k, m}, \quad 0 \leqslant k \leqslant p-s-1, \quad 0 \leqslant m \leqslant r \text {, } \\
& K \mathrm{~b}_{n, m}= \pm \mathfrak{q}^{s-1-2 n} \mathrm{~b}_{n, m}, \quad 0 \leqslant n \leqslant s-1, \quad 0 \leqslant m \leqslant r-1, \\
& E \mathrm{t}_{n, m}=\left\{\begin{array}{ll} 
\pm[n][s-n] \mathrm{t}_{n-1, m} \pm g \mathrm{~b}_{n-1, m}, & 1 \leqslant n \leqslant s-1, \\
\pm g \frac{r-m}{r} \mathrm{r}_{p-s-1, m} \pm\left. g \frac{m}{r}\right|_{p-s-1, m}, & n=0,
\end{array} \quad 0 \leqslant m \leqslant r-1,\right. \\
& \left.E\right|_{k, m}=\left\{\begin{array}{ll}
\left.\mp[k][p-s-k]\right|_{k-1, m}, & 1 \leqslant k \leqslant p-s-1, \\
\pm g(m-r) \mathrm{b}_{s-1, m-1}, & k=0,
\end{array} \quad 1 \leqslant m \leqslant r-1,\right. \\
& \operatorname{Er}_{k, m}=\left\{\begin{array}{ll}
\mp[k][p-s-k] \mathrm{r}_{k-1, m}, & 1 \leqslant k \leqslant p-s-1, \\
\pm g m \mathrm{~b}_{s-1, m-1}, & k=0,
\end{array} \quad 0 \leqslant m \leqslant r,\right. \\
& E \mathrm{~b}_{n, m}= \pm[n][s-n] \mathrm{b}_{n-1, m}, \quad 1 \leqslant n \leqslant s-1, \quad 0 \leqslant m \leqslant r-1 \quad\left(\mathrm{~b}_{-1, m} \equiv 0\right) \text {, } \\
& F \mathrm{t}_{n, m}= \begin{cases}\mathrm{t}_{n+1, m}, & 0 \leqslant n \leqslant s-2, \\
\frac{1}{r} \mathrm{r}_{0, m+1}-\frac{1}{r} \mathrm{I}_{0, m+1}, & n=s-1 \quad\left(\mathrm{I}_{0, r} \equiv 0\right),\end{cases} \\
& F \mathrm{I}_{k, m}=\left\{\begin{array}{ll}
\mathrm{l}_{k+1, m}, & 0 \leqslant k \leqslant p-s-2, \\
\mathrm{~b}_{0, m}, & k=p-s-1,
\end{array} \quad 1 \leqslant m \leqslant r-1,\right. \\
& F \mathrm{r}_{k, m}=\left\{\begin{array}{ll}
\mathrm{r}_{k+1, m}, & 0 \leqslant k \leqslant p-s-2, \\
\mathrm{~b}_{0, m}, & k=p-s-1,
\end{array} \quad 0 \leqslant m \leqslant r,\right. \\
& F \mathrm{~b}_{n, m}=\mathrm{b}_{n+1, m}, \quad 1 \leqslant n \leqslant s-1, \quad 0 \leqslant m \leqslant r-1 \quad\left(\mathrm{~b}_{s, m} \equiv 0\right) \text {. }
\end{aligned}
$$

where $g=\frac{(-1)^{p}[s]}{[p-1] !}$.

In thus introduced basis, the $s \ell(2)$-generators $e, f$ and $h$ act in $\mathcal{P}_{s, r}^{ \pm}$as in the direct sum $X_{s, r}^{\alpha} \oplus X_{p-s, r-1}^{-\alpha} \oplus X_{p-s, r+1}^{-\alpha} \oplus X_{s, r}^{\alpha}$ (see (3.1)-(3.3) $)$, where for $r=1$ we set $X_{p-s, 0}^{-\alpha} \equiv 0$.

\section{REFERENCES}

[1] P.A. Pearce, J. Rasmussen, J.-B. Zuber, Logarithmic Minimal Models, J.Stat.Mech. 0611 (2006) P017, hep-th/0607232

[2] N. Read and H. Saleur, Associative-algebraic approach to logarithmic conformal field theories, Nucl. Phys. B 777, 316 (2007) [arXiv:hep-th/0701117|. 
[3] P.A. Pearce, J. Rasmussen, P. Ruelle, Integrable Boundary Conditions and W-Extended Fusion in the Logarithmic Minimal Models LM(1,p), arXiv:0803.0785

[4] J. Rasmussen, P.A. Pearce, W-Extended Fusion Algebra of Critical Percolation, arXiv:0804.4335

[5] J.Rasmussen, W-Extended Logarithmic Minimal Models, arXiv:0805.2991.

[6] B.L. Feigin, A.M. Gainutdinov, A.M. Semikhatov, and I.Yu. Tipunin, Logarithmic extensions of minimal models: characters and modular transformations, Nucl.Phys. B757 (2006) 303-343, hep-th/0606196

[7] H.G. Kausch, Extended conformal algebras generated by a multiplet of primary fields, Phys. Lett. B 259 (1991) 448.

[8] J. Fuchs, S. Hwang, A.M. Semikhatov, and I.Yu. Tipunin, Nonsemisimple fusion algebras and the Verlinde formula, Commun. Math. Phys. 247 (2004) 713-742 [hep-th/0306274].

[9] H.G. Kausch, Symplectic Fermions, Nucl.Phys. B583 (2000) 513-541.

[10] M.R. Gaberdiel and H.G. Kausch, A rational logarithmic conformal field theory, Phys. Lett. B 386 (1996) 131 [hep-th/9606050].

[11] M.Flohr, On Fusion Rules in Logarithmic Conformal Field Theories, Int.J.Mod.Phys. A12 (1997) 1943-1958, arXiv:hep-th/9605151.

[12] M.R. Gaberdiel and H.G. Kausch, A local logarithmic conformal field theory, Nucl. Phys. B538 (1999) 631-658 [hep-th/9807091].

[13] M.Flohr, On Modular Invariant Partition Functions of Conformal Field Theories with Logarithmic Operators, Int.J.Mod.Phys. A11 (1996) 4147-4172, arXiv:hep-th/9509166

[14] M.R. Gaberdiel, An algebraic approach to logarithmic conformal field theory, Int. J. Mod. Phys. A18 (2003) 4593-4638 [hep-th/0111260].

[15] M. Flohr, Bits and pieces in logarithmic conformal field theory, Int. J. Mod. Phys. A18 (2003) 44974592 [hep-th/0111228].

[16] M.R. Gaberdiel and I. Runkel, The logarithmic triplet theory with boundary, J. Phys. A 39 (2006) 14745, hep-th/0608184.

[17] M.R. Gaberdiel and I. Runkel, From boundary to bulk in logarithmic CFT, arXiv:0707.0388 [hep-th].

[18] A.M. Gainutdinov and I.Yu. Tipunin, Radford, Drinfeld, and Cardy boundary states in $(1, p)$ logarithmic conformal field models, arXiv:0711.3430.

[19] B.L. Feigin, A.M. Gainutdinov, A.M. Semikhatov, and I.Yu. Tipunin, Modular group representations and fusion in logarithmic conformal field theories and in the quantum group center, Commun. Math. Phys. 265 (2006) 47-93. [hep-th/0504093].

[20] B.L. Feigin, A.M. Gainutdinov, A.M. Semikhatov, and I.Yu. Tipunin, Kazhdan-Lusztig correspondence for the representation category of the triplet $W$-algebra in logarithmic CFT, Theor. Math. Phys. 148 (2006) 1210-1235. [math.QA/0512621].

[21] A.M. Semikhatov, Factorizable ribbon quantum groups in logarithmic conformal field theories, Theor.Math.Phys.154: 433-453, 2008; [arXiv:0705.4267].

[22] B.L. Feigin, A.M. Gainutdinov, A.M. Semikhatov, and I.Yu. Tipunin, Kazhdan-Lusztig-dual quantum group for logarithmic extensions of Virasoro minimal models, J. Math. Phys. 48 (2007) 032303, [math.QA/0606506].

[23] J. Rasmussen, Polynomial fusion rings of W-extended logarithmic minimal models, arXiv:0812.1070,

[24] A.M. Semikhatov, A note on the logarithmic ( $\left.p, p^{\prime}\right)$ fusion, arXiv:0710.5157 [hep-th].

[25] J. Rasmussen and P. A. Pearce, Fusion Algebras of Logarithmic Minimal Models, J. Phys. A 40, 13711 (2007) [arXiv:0707.3189 [hep-th]].

[26] H. Eberle, M. Flohr, Virasoro representations and fusion for general augmented minimal models, J.Phys.A39:15245-15286,2006. 
[27] N. Carqueville, M. Flohr, Nonmeromorphic operator product expansion and $C_{2}$-cofiniteness for a family of W-algebras, J.Phys. A39 (2006) 951-966

[28] M. Flohr, C. Grabow, M. Koehn, Fermionic Expressions for the Characters of c(p,1) Logarithmic Conformal Field Theories, Nucl.Phys.B768:263-276,2007

[29] M. Flohr, H. Knuth, On Verlinde-Like Formulas in $c_{p, 1}$ Logarithmic Conformal Field Theories, arXiv:0705.0545

[30] N. Read, H. Saleur, Enlarged symmetry algebras of spin chains, loop models, and S-matrices, Nucl.Phys.B777:263-315,2007.

[31] A. Dossavitsky, B.L. Feigin, A.M. Gainutdinov, I.Yu. Tipunin, in preparation.

[32] S. MacLane, Homology, Springer-Verlag, 1963.

[33] K. Erdmann, E.L. Green, N. Snashall, R. Taillefer, Representation theory of the Drinfel'd doubles of a family of Hopf algebras, math-rt/0410017.

[34] P.V. Bushlanov, B.L. Feigin, A.M. Gainutdinov, I.Yu. Tipunin, in preparation.

[35] B.L. Feigin, I.Yu. Tipunin, Characters of coinvariants in (1,p) logarithmic models, arXiv:0805.4096.

[36] M.R. Gaberdiel and H.G. Kausch, Indecomposable fusion products, Nucl. Phys. B477 293-318 (1996).

PVB:Moscow Institute of Physics and Technology, Dolgoprudny, Moskovskoe shosse 21A , RUSSIA, 141700

E-mail address: paulbush@mail.ru

BLF:Higher School of Economics, Moscow, Russia and Landau institute For THeORetical Physics, Chernogolovka, 142432, Russia

E-mail address: bfeiginegmail.com

Amg:Tamm Theory Division, Lebedev Physics Institute, Leninski PR., 53, Moscow, RUSSIA, 119991

E-mail address: gainut@gmail.com

IYut:Tamm Theory Division, Lebedev Physics Institute, Leninski PR., 53, Moscow, RUSSIA, 119991

E-mail address: tipuninegmail.com 